\title{
ASSESSMENT OF RAINFALL EROSIVITY FACTOR (R) FOR SELECTED LOCATIONS IN IMO STATE, SOUTHEASTERN NIGERIA
}

\author{
OKORAFOR, O.O, EGWUONWU, C.C \\ Department of Agricultural and Bioresources Engineering, \\ Federal University of Technology, Owerri, Imo State, Nigeria. \\ AKINBILE, C.O \\ Department of Agricultural and Environmental Engineering, \\ Federal University of Technology, Akure, Ondo State, Nigeria. \\ ADEYEMO, A.J \\ Department of Crop, Soil and Pest Management, \\ Federal University of Technology, Akure Ondo State, Nigeria.
}

\begin{abstract}
Soil erosion in southeastern Nigeria is a major environmental issue of which rainfall is a major contributor to its elevated level(s). This study gives a critical assessment on the rainfall erosivity factor $(R)$ for selected sites in Imo State representing different soil groups geologically by making use of mean monthly rainfall data and GIS techniques. By applying empirical methods the rainfall intensity for all the locations were obtained and was further determined at three different intervals of 30-minutes, 45minutes and 60-minutes respectively. Through the use of the revised universal soil loss equation (RUSLE) the rainfall erosivity factor $(\mathbf{R})$ was obtained for each of the locations and they are as follows; $6054.93 \mathrm{MJmm} / \mathrm{ha} / \mathrm{hr}, 5211.48$ $\mathrm{MJmm} / \mathrm{ha} / \mathrm{hr}$, 5977.29 MJmm/ha/hr, 6171.57 MJmm/ha/hr, 6478.06 MJmm/ha/hr, 5567.54 MJmm/ha/hr, 5350.84 $\mathrm{MJmm} / \mathrm{ha} / \mathrm{hr}$ and 5470.29 MJmm/ha/hr for Ehime Mbano, Ideato North, Ikeduru, Oguta, Ohajiegbema, Orlu and Owerri West respectively. Likewise the rainfall erosivity factor $(\mathbf{R})$ was determined at different rainfall intensities and it was observed that the factor $(R)$ reduced with increase in rainfall intensity, thus conforming to the fact that as rainfall duration increases the soil approaches saturation and erosive force reduces as well. Also, the rainfall erosivity factor $(R)$ for all the locations were subjected to a correlation analysis and they all yielded a strong/perfect correlation with a correlation coefficient of about 0.95 which shows or implies $95 \%$ strength. On further subjection to ANOVA the rainfall erosivity factor (R) for all the locations showed statistical significance at 5\% value thus showing uniformity of region and non-variation in rainfall characteristics. The values obtained for each of the locations were mapped also to show the varying degree in the factor. Though rainfall is a natural climatic factor which is not amenable to human interference, it is therefore
\end{abstract}

important to enact and adopt proper cover/management practices/measures so as to sustain the environment against further effects.

Keywords: Soil, Erosion, Erosivity, RUSLE, Rainfall Erosivity Factor

\section{INTRODUCTION}

Agricultural soils are the richest supplies of minerals, nutrients, moisture, air, organic materials and microbial activities that support plant growth and production. They provide the pathways through which water and nutrients move to the roots of plants, and they are the matrix of nutrient transformation with the resultant environment for micro-organisms and fauna (Powlson et al., 2011). The availability of soils for agricultural production and food security is limited by the irreparable/destructive effects of erosion. Soil erosion is a serious environmental, economic and social problem, which causes severe land degradation, reduced productivity levels and threatens the stability and health of society in general alongside sustainable development of rural areas (Wang et al., 2013).Soil erosion is the gradual or rapid weathering/disintegration, removal and transportation of soil aggregates through the action of erosive agents such as wind, water, gravity and human disturbance (Gunawan et al., 2013). Soil loss by runoff (overland flow caused by rainfall) is a severe ecological problem occupying $56 \%$ of the world wide area and is accelerated by human induced soil degradation (Gelagay and Minale, 2016; Bai et al., 2008). Also the extent of deterioration of soils and water resources due to erosion and pollution has assumed a frightening dimension with its attendant effects on global food security, water quality and hygiene for sustainable livelihoods (Akinbile et al., 2016). The impact of soil erosion is felt in the inability of the soil to meet up with the moisture and nutrient requirements of the plants thereby resulting into soil 
degradation and low agricultural productivity. According to Michael and Ojha (2003), the potential for erosion is dependent on certain factors which include soil nature/characteristics, slope/topography, presence of vegetative cover and general climatic conditions. The major contributing factor to soil loss and movement is rainfall and its characteristics (intensity, duration, frequency, energy, distribution e.t.c) (Okorafor et al., 2017). The erosive ability of rainfall to initiate soil detachment, movement/transportation and eventual deposition in different locations is regarded as the erosivity of rainfall. Rainfall erosivity factor $(\mathrm{R})$ is one of the six factors of the Universal Soil Loss Equation (Wischmeier and Smith, 1978) and Revised Universal Soil Loss Equation (Renard et al., 1997) which is stated thus;

$A=R K S C P$

\section{Where $A=$ Annual soil Loss \\ $\mathrm{R}=$ Rainfall Erosivity Factor \\ $\mathrm{K}=$ Soil Erodibility Factor \\ $\mathrm{S}=$ Slope Length and Steepness Factor \\ $\mathrm{C}=$ Cover Crop Management Factor \\ $\mathrm{P}=$ Conservation/Management Practice Factor}

Rainfall erosivity factor is dependent on the amount, duration, intensity, raindrop size, distribution, frequency and kinetic energy of a rainfall/storm event (Okorafor et al., 2017). This is a natural factor since it is not amenable to human modification and cannot be adjusted or regulated to prevent the resultant damage it may cause. The main objective of this study is to make use of mean monthly rainfall data to determine rainfall erosivity factor (R) for Imo State (different soil groups/locations) and to make use of geographical information system (GIS) to map the study area according to the magnitude of the rainfall erosivity factor $(\mathrm{R})$.

\section{MATERIALS AND METHODS}

\subsection{Description of Study Area}

Imo State is located within latitude $4^{\circ} 45^{\prime} \mathrm{N}$ and $7^{\circ} 15^{\prime} \mathrm{N}$ and longitude $6^{\circ} 50^{\prime} \mathrm{E}$ and $7^{\circ} 25^{\prime} \mathrm{E}$ and covers an area of about 5100 $\mathrm{km}^{2}$ and falls within the rain forest belt of Nigeria with two distinct seasons (i.e. wet and dry) which exists within the region (Okoro et al., 2014; Okorie, 2015). The study area experiences high rainfall amounts of about 1600-2900 mm (Selemo et al., 2012; Okorafor et al., 2018). The relative humidity ranges from 75-90\% (Udokporo et al., 2015) with mean minimum temperature of $23.5^{\circ} \mathrm{C}$ and mean maximum temperature of $32.9^{\circ} \mathrm{C}$ (Okorie, 2015). According to Nkheloane et al., 2012; Ernest and Onweremadu, 2016), the soils of Imo State have a characterized brown colour ranging from strong dark brown to yellowish red thus indicating the strong presence of iron oxide; also soils of Imo State are characterized by high sand percentage, low organic matter content and low water storage capacity thus placing the zone as an area of high susceptibility to accelerated erosion and land degradation (Ufot et al., 2016: Ibeje, 2016). The soils are predominantly ultisols derived from coastal plain sand (benin formation) and have low $\mathrm{pH}$ with exchangeable bases (Onwudike, 2010). The high temperature and humidity experienced within the region favors luxuriant plant growths which produce the climax vegetation of the tropical rainforest zone (Udokporo et al., 2015). Furthermore the watershed is covered by depleted rainforest shrubs having thick under brushes, creeping vines and deep green vegetation which is as a result of heavy decay of plant droppings, foliage and litter. The map of Imo State is as shown in Figure 1.

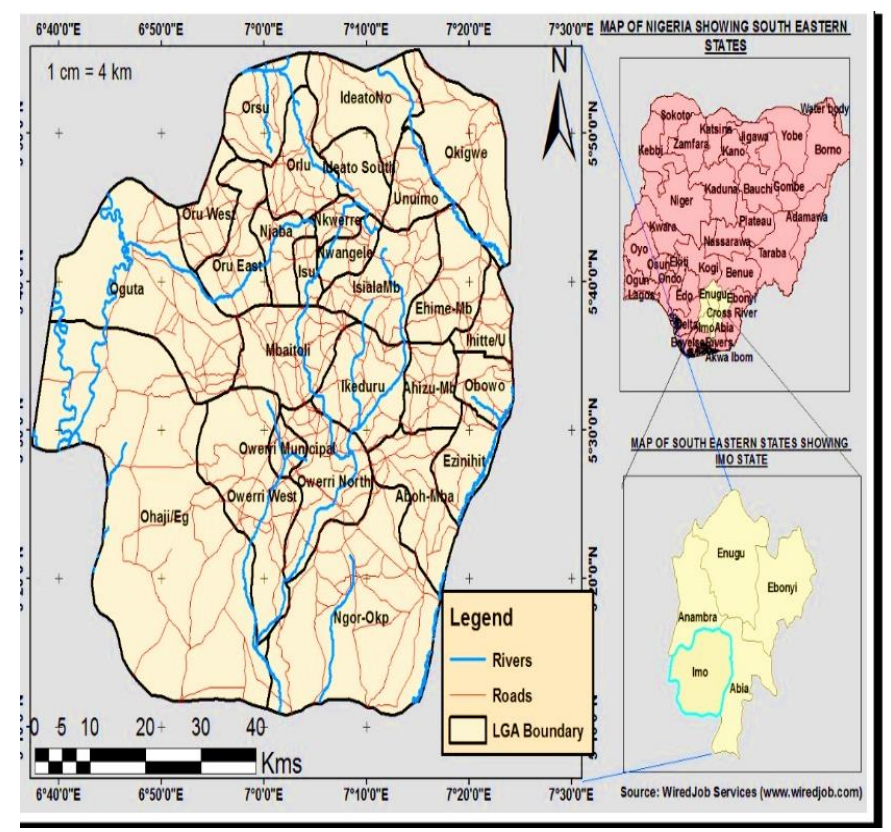

Figure 1: Map of Study Area (Okorafor et al., 2018)

\subsection{Selection of Locations}

The study area was subdivided into eight soil groups based on geological classification of base materials found within each zone. The various soil groups with their major towns and percentage area of coverage are as summarized in Table 1. The soil map also indicating the various soil groups are as shown in Figure 2.

Table 2.1: Soil Groups, Percentage Coverage and Site Locations

\begin{tabular}{|l|c|l|}
\hline \multicolumn{1}{|c|}{ Soil Type/Group } & $\begin{array}{l}\text { Percentage } \\
\text { Coverage }\end{array}$ & Site Location \\
\hline $\begin{array}{l}\text { Sands, gravel and clay } \\
\text { (Meander belts). }\end{array}$ & 5.9 & OhajiEgbema \\
\hline $\begin{array}{l}\text { Sands and clay (Coastal plains } \\
\text { sands). }\end{array}$ & 48.6 & Owerri West \\
\hline $\begin{array}{l}\text { Sands, clays and swamps } \\
\text { (Sombreiro Deltaic plane) }\end{array}$ & 1.8 & Oguta \\
\hline $\begin{array}{l}\text { Clays, sandstones, lignite and } \\
\text { shales (Lignite formation) }\end{array}$ & 12.2 & Ikeduru \\
\hline $\begin{array}{l}\text { Clayey sands and shales } \\
\text { (Bende-Ameke and Nnanka } \\
\text { stones). }\end{array}$ & 20.3 & Orlu \\
\hline $\begin{array}{l}\text { Clay and shales (Ebenebe and } \\
\text { Umuna sandstones). }\end{array}$ & 6.3 & Ehime Mbano \\
\hline
\end{tabular}




\begin{tabular}{|l|l|l|}
\hline Sandstones (Imo formation). & 1.2 & Ideato North \\
\hline $\begin{array}{l}\text { Sandstones, Limestones and } \\
\text { Coal(Upper coal). }\end{array}$ & 3.6 & Okigwe \\
\hline
\end{tabular}
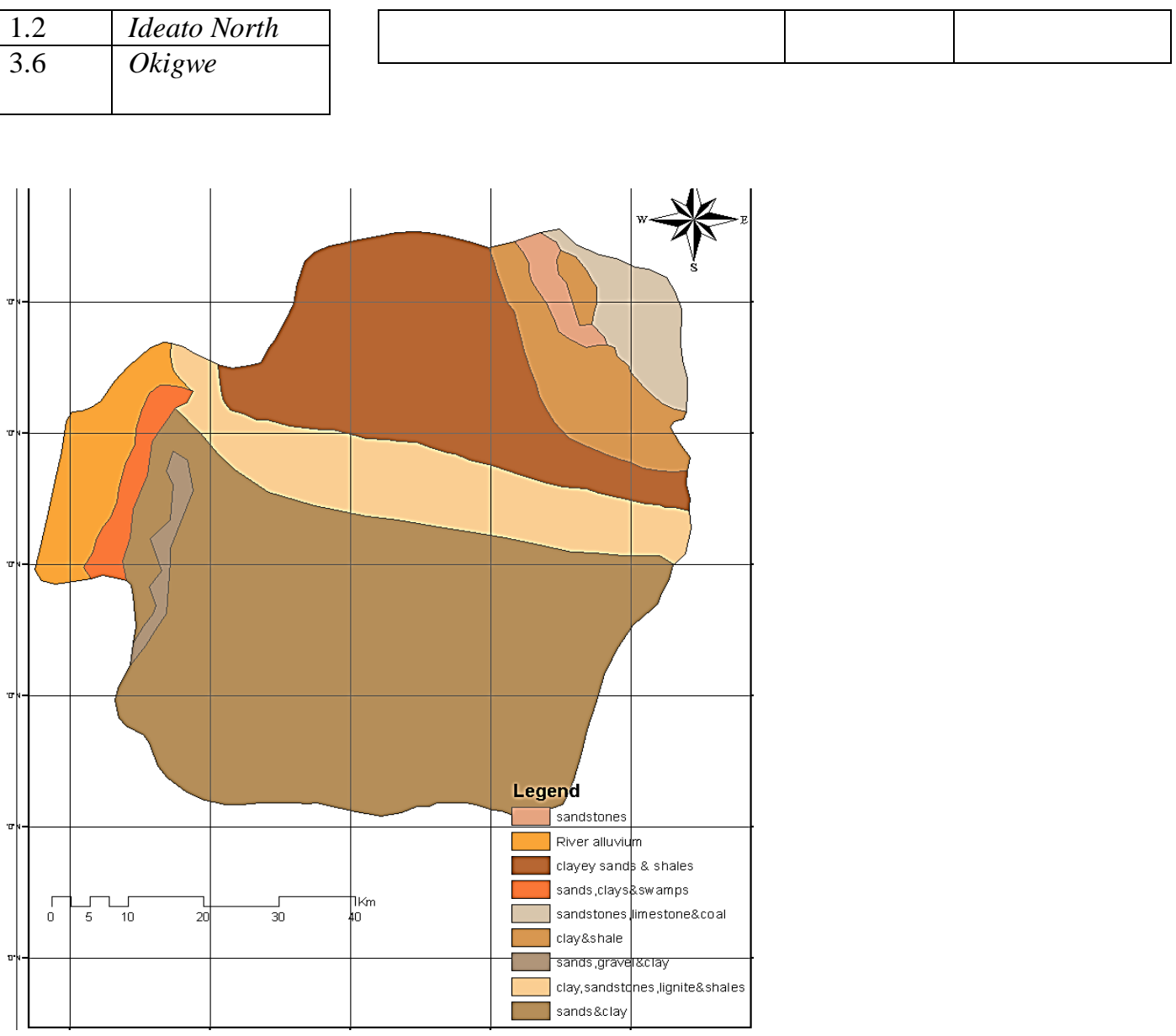

Figure 2: Soil Map of the Study Area (Okorafor et al., 2018)

\subsection{Data Collection and Computation}

In order to determine the rainfall erosivity factor $(\mathrm{R})$ of the selected locations rainfall data (Mean monthly rainfall amount and rainfall days) were obtained from NIMET (Nigerian Meteorological Agency) for a period of 31 years (1986-2016). According to Sanchez-Moreno et al, (2013); Oliviera et al, (2012) rainfall erosivity factor $(\mathrm{R})$ is computed thus;

$R=\Sigma\left(E I_{30}\right)$.

Where $\mathrm{R}=$ Rainfall Erosivity Factor

$\mathrm{I}_{30}=30$ minutes rainfall intensity

$\mathrm{E}=$ Total Storm Kinetic Energy

The kinetic energy of the storm or rainfall event is obtained from the following relationship according to Teh (2011) as;

$E=210.3+87 \log _{10} I$.

Where $\mathrm{E}=$ Unitary kinetic energy

$$
\mathrm{I}=\text { Rainfall Intensity }
$$

The rainfall intensity (I) which is the rate of rainfall express as depth per time (Michael and Ojha, 2003) is given by Yin et al, (2007) as;

$I=P / T$
Where $\mathrm{P}=$ Precipitation

$$
\mathrm{T}=\text { Duration of Rainfall }
$$

The resultant 30 minute interval rainfall $\left(\mathrm{I}_{30}\right)$ is also obtained according to Yin et al, (2007) as;

$I_{30}=P_{30} / 0.5 h$

Where $\mathrm{P}_{30}=$ Maximum 30 minute rainfall

$0.5 \mathrm{~h}=30$ minutes duration

The results obtained for the rainfall erosivity factor $(\mathrm{R})$ for all the locations representing different soil groups was interfaced with ArcGis 10.1 and under raster distribution the study area was mapped to reveal the variation of rainfall erosivity factor (R) within the various soil groups.

\section{RESULTS}

Based on the 31year rainfall data used rainfall intensity for different time intervals (30minutes, 45minutes and 60minutes) was obtained and based on the varying time intervals the kinetic energy and rainfall erosivity factor $(\mathrm{R})$ were also obtained for all the various locations representing different soil groups as well. The results for rainfall intensity, kinetic energy and 
International Journal of Engineering Applied Sciences and Technology, 2019

Vol. 4, Issue 6, ISSN No. 2455-2143, Pages 319-332

Published Online October 2019 in IJEAST (http://www.ijeast.com)

rainfall erosivity factor $(\mathrm{R})$ for each location are as shown in

Tables 3.1-3.9. The summary of the mean rainfall erosivity factor (R) i.e $\mathrm{EI}_{30}$ for all the locations (different soil groups) are also as shown in Table 3.10 .

Table 3.1: Mean Rainfall Amount and Rainfall Days for all the Locations (1986- 2016)

\begin{tabular}{|l|c|c|c|c|c|c|c|c|c|}
\hline Month & $\begin{array}{c}\text { Ehime } \\
\text { Mbano } \\
(\mathbf{m m})\end{array}$ & $\begin{array}{c}\text { Ideato } \\
\text { North } \\
(\mathbf{m m})\end{array}$ & $\begin{array}{c}\text { Ikeduru } \\
(\mathbf{m m})\end{array}$ & $\begin{array}{c}\text { Oguta } \\
(\mathbf{m m})\end{array}$ & $\begin{array}{c}\text { Ohaji } \\
(\mathbf{m m})\end{array}$ & $\begin{array}{c}\text { Okigwe } \\
(\mathbf{m m})\end{array}$ & $\begin{array}{c}\text { Orlu } \\
(\mathbf{m m})\end{array}$ & $\begin{array}{c}\text { Owerri } \\
\text { West } \\
(\mathbf{m m})\end{array}$ & $\begin{array}{c}\text { Rainfall } \\
\text { Days } \\
(\mathbf{h r s})\end{array}$ \\
\hline January & 110 & 90 & 90 & 130 & 110 & 100 & 110 & 8 & 24.72 \\
\hline February & 520 & 370 & 390 & 410 & 450 & 430 & 360 & 300 & 35.52 \\
\hline March & 1100 & 980 & 1120 & 1000 & 1220 & 1030 & 950 & 1090 & 98.40 \\
\hline April & 1460 & 1470 & 1490 & 1580 & 1680 & 1420 & 1510 & 1560 & 165.6 \\
\hline May & 2830 & 2800 & 2700 & 2850 & 2930 & 2790 & 2700 & 2670 & 241.68 \\
\hline June & 3200 & 2920 & 3610 & 3520 & 3880 & 2980 & 2860 & 4070 & 263.28 \\
\hline July & 3550 & 3120 & 3470 & 4090 & 3500 & 3310 & 3370 & 3030 & 334.56 \\
\hline August & 3320 & 3030 & 3600 & 3150 & 3410 & 3400 & 3070 & 3990 & 349.92 \\
\hline September & 3210 & 3080 & 3110 & 3640 & 3500 & 3100 & 3280 & 2760 & 337.68 \\
\hline October & 2610 & 2490 & 2680 & 2900 & 2830 & 2570 & 2550 & 2790 & 274.8 \\
\hline November & 740 & 620 & 750 & 790 & 840 & 710 & 650 & 680 & 74.4 \\
\hline December & 110 & 70 & 120 & 90 & 120 & 80 & 80 & 14 & 12.48 \\
\hline
\end{tabular}

Table 3.2: Rainfall Intensity (I), Rainfall Energy (E) and Rainfall Erosivity Factor (R) for Ehime Mbano (Clay and Shales)

\begin{tabular}{|c|c|c|c|c|c|c|c|}
\hline Month & $\begin{array}{c}\mathbf{I}_{30} \\
(\mathbf{m m} / \mathbf{h r})\end{array}$ & $\begin{array}{c}\mathbf{I}_{45} \\
(\mathrm{~mm} / \mathrm{hr})\end{array}$ & $\begin{array}{c}\mathbf{I}_{60} \\
(\mathrm{~mm} / \mathrm{hr})\end{array}$ & $\begin{array}{c}\mathrm{E} \\
(\mathbf{M J m m} / \mathbf{h a})\end{array}$ & $\begin{array}{c}\mathbf{E I}_{30}(\mathbf{R}) \\
\left(\mathbf{M J m m} / \mathbf{h a h r}^{-1}\right)\end{array}$ & $\begin{array}{c}\text { EI }_{45}(\mathbf{R}) \\
\left(\mathbf{M J m m} / \mathbf{h a h r}^{-1}\right)\end{array}$ & $\begin{array}{c}\text { EI }_{60}(\mathbf{R}) \\
\left(\mathbf{M J m m} / \mathbf{h a h r}^{-1}\right)\end{array}$ \\
\hline January & 8.899 & 5.933 & 4.449 & 267.995 & 2381.95 & 1590.01 & 1192.31 \\
\hline February & 29.279 & 19.519 & 14.639 & 314.030 & 9202.39 & 6129.55 & 4597.09 \\
\hline March & 22.358 & 14.905 & 11.179 & 303.507 & 6788.05 & 4525.26 & 3394.02 \\
\hline April & 17.633 & 11.755 & 8.816 & 294.429 & 5191.67 & 3461.01 & 2595.69 \\
\hline May & 23.427 & 15.612 & 11.709 & 305.398 & 7154.56 & 4767.09 & 3575.32 \\
\hline June & 24.309 & 16.456 & 12.154 & 306.840 & 7458.97 & 5049.34 & 3729.33 \\
\hline July & 21.222 & 14.147 & 10.611 & 301.592 & 6400.39 & 4266.62 & 3200.19 \\
\hline August & 18.976 & 12.651 & 9.488 & 297.268 & 5640.96 & 3760.74 & 2820.48 \\
\hline September & 19.012 & 12.675 & 9.506 & 297.341 & 5653.05 & 3768.79 & 2826.52 \\
\hline October & 18.996 & 12.664 & 9.498 & 297.309 & 5647.68 & 3765.12 & 2823.84 \\
\hline November & 19.892 & 13.262 & 9.946 & 299.091 & 5949.52 & 3966.55 & 2974.76 \\
\hline December & 17.628 & 11.752 & 8.814 & 294.420 & 5190.04 & 3460.02 & 2595.02 \\
\hline
\end{tabular}


International Journal of Engineering Applied Sciences and Technology, 2019

Vol. 4, Issue 6, ISSN No. 2455-2143, Pages 319-332

Published Online October 2019 in IJEAST (http://www.ijeast.com)

Table 3.3: Rainfall Intensity (I), Rainfall Energy (E) and Rainfall Erosivity Factor (R) for Ideato North (Sandstones)

\begin{tabular}{|c|c|c|c|c|c|c|c|}
\hline Month & $\begin{array}{c}\mathbf{I}_{30} \\
(\mathbf{m m} / \mathbf{h r})\end{array}$ & $\begin{array}{c}\mathbf{I}_{45} \\
(\mathrm{~mm} / \mathrm{hr})\end{array}$ & $\begin{array}{c}\mathbf{I}_{60} \\
(\mathbf{m m} / \mathbf{h r})\end{array}$ & $\begin{array}{c}E \\
(\mathbf{M J m m} / \mathbf{h a})\end{array}$ & $\begin{array}{c}\mathbf{E I}_{30}(\mathbf{R}) \\
\left(\mathbf{M J m m} / \mathbf{h a h r}^{-1}\right)\end{array}$ & $\begin{array}{c}\text { EI }_{45}(\mathbf{R}) \\
\left(\mathbf{M J m m} / \mathbf{h a h r}^{-1}\right)\end{array}$ & $\begin{array}{c}\text { EI }_{60}(\mathbf{R}) \\
\left(\mathrm{MJmm} / \mathrm{hahr}^{-1}\right)\end{array}$ \\
\hline Jan. & 7.282 & 4.854 & 3.641 & 260.25 & 1895.14 & 1263.25 & 947.57 \\
\hline Feb. & 20.833 & 13.889 & 10.417 & 300.88 & 6288.23 & 4178.92 & 3134.27 \\
\hline Mar. & 19.919 & 13.279 & 9.959 & 299.14 & 5958.57 & 3972.28 & 2979.14 \\
\hline Apr. & 17.754 & 11.836 & 8.877 & 294.69 & 5231.93 & 3487.95 & 2615.96 \\
\hline May & 23.179 & 15.447 & 11.586 & 304.99 & 7069.37 & 4711.18 & 3533.61 \\
\hline Jun. & 22.182 & 15.016 & 11.091 & 303.30 & 6727.80 & 4554.35 & 3363.90 \\
\hline Jul. & 18.651 & 12.434 & 9.326 & 296.60 & 5531.89 & 3687.92 & 2766.09 \\
\hline Aug. & 17.318 & 11.546 & 8.659 & 293.74 & 5086.99 & 3391.52 & 2543.49 \\
\hline Sept & 18.242 & 12.161 & 9.121 & 295.74 & 5394.89 & 3596.49 & 2697.45 \\
\hline Oct. & 18.122 & 12.081 & 9.061 & 295.49 & 5354.87 & 2569.82 & 2677.43 \\
\hline Nov. & 16.667 & 11.111 & 8.333 & 292.25 & 4870.93 & 3247.19 & 2435.32 \\
\hline Dec. & 11.218 & 7.48 & 5.609 & 276.95 & 3106.83 & 2071.59 & 1553.41 \\
\hline
\end{tabular}

Table 3.4: Rainfall Intensity (I), Rainfall Energy (E) and Rainfall Erosivity Factor (R) for Ikeduru (Clays, Sandstones, Lignite and Shales)

\begin{tabular}{|c|c|c|c|c|c|c|c|}
\hline Month & $\begin{array}{c}\mathbf{I}_{30} \\
(\mathbf{m m} / \mathbf{h r})\end{array}$ & $\begin{array}{c}\mathbf{I}_{45} \\
(\mathrm{~mm} / \mathrm{hr})\end{array}$ & $\begin{array}{c}\mathbf{I}_{60} \\
(\mathrm{~mm} / \mathrm{hr})\end{array}$ & $\begin{array}{c}E \\
(\mathbf{M J m m} / \mathbf{h a})\end{array}$ & $\begin{array}{c}\text { EI I0 }_{30}(R) \\
\left(\mathbf{M J m m} / \mathbf{h a h r}^{-1}\right)\end{array}$ & $\begin{array}{c}\mathbf{E I}_{45}(\mathbf{R}) \\
\left(\mathbf{M J m m} / \mathbf{h a h r}^{-1}\right)\end{array}$ & $\begin{array}{c}\text { EI }_{60}(\mathbf{R}) \\
\left(\mathbf{M J m m} / \mathbf{h a h r}^{-1}\right)\end{array}$ \\
\hline January & 7.282 & 4.854 & 3.641 & 260.25 & 1895.14 & 1263.25 & 947.57 \\
\hline February & 21.959 & 14.639 & 10.979 & 302.91 & 6651.60 & 4434.30 & 3325.65 \\
\hline March & 22.764 & 15.176 & 11.382 & 304.30 & 6927.09 & 4618.06 & 3463.54 \\
\hline April & 17.995 & 11.997 & 8.998 & 295.22 & 5312.48 & 3541.75 & 2656.39 \\
\hline May & 22.351 & 14.896 & 11.172 & 303.58 & 6839.96 & 4522.13 & 3391.60 \\
\hline June & 27.423 & 18.564 & 13.712 & 311.50 & 8542.27 & 5782.69 & 4271.29 \\
\hline July & 20.744 & 13.829 & 10.372 & 300.71 & 6337.95 & 4158.52 & 3118.97 \\
\hline August & 20.576 & 13.717 & 10.288 & 300.39 & 6180.83 & 4120.45 & 3090.41 \\
\hline September & 18.419 & 12.279 & 9.209 & 296.12 & 5454.23 & 3636.06 & 2726.97 \\
\hline October & 19.505 & 13.003 & 9.753 & 298.33 & 5818.93 & 3879.19 & 2909.61 \\
\hline November & 20.161 & 13.441 & 10.081 & 299.61 & 6040.44 & 4027.06 & 3020.37 \\
\hline December & 19.231 & 12.821 & 9.615 & 297.78 & 5726.61 & 3817.84 & 2863.16 \\
\hline
\end{tabular}


International Journal of Engineering Applied Sciences and Technology, 2019

Vol. 4, Issue 6, ISSN No. 2455-2143, Pages 319-332

Published Online October 2019 in IJEAST (http://www.ijeast.com)

Table 3.5: Rainfall Intensity (I), Rainfall Energy (E) and Rainfall Erosivity Factor (R) for Oguta (Sands, Clays and Swamps)

\begin{tabular}{|c|c|c|c|c|c|c|c|}
\hline Months & $\begin{array}{c}\mathbf{I}_{30} \\
(\mathrm{~mm} / \mathrm{hr})\end{array}$ & $\begin{array}{c}\mathbf{I}_{45} \\
(\mathrm{~mm} / \mathrm{hr})\end{array}$ & $\begin{array}{c}\mathbf{I}_{60} \\
(\mathrm{~mm} / \mathrm{hr})\end{array}$ & $\begin{array}{c}\mathbf{E} \\
(\mathbf{M J m m} / \mathbf{h a})\end{array}$ & $\begin{array}{c}\mathrm{EI}_{30}(\mathbf{R}) \\
\left(\mathbf{M J m m} / \mathbf{h a h r}^{-1}\right)\end{array}$ & $\begin{array}{c}\mathbf{E I}_{45} \\
\left(\mathbf{M J m m} / \mathrm{hahr}^{-1}\right)\end{array}$ & $\begin{array}{c}\text { EI }_{60} \\
\left(\mathbf{M J m m} / h^{2} r^{-1}\right)\end{array}$ \\
\hline January & 10.518 & 7.012 & 5.259 & 274.46 & 2886.77 & 1924.51 & 1517.49 \\
\hline February & 23.086 & 15.390 & 11.543 & 304.85 & 7037.77 & 4691.64 & 3518.88 \\
\hline March & 20.325 & 13.550 & 10.162 & 299.92 & 6095.87 & 4063.92 & 3047.79 \\
\hline April & 19.082 & 12.721 & 9.541 & 297.48 & 5676.51 & 3784.24 & 2830.22 \\
\hline May & 23.593 & 15.723 & 11.793 & 305.68 & 7211.91 & 4806.21 & 3604.88 \\
\hline June & 26.739 & 18.101 & 13.369 & 310.52 & 8302.99 & 5620.72 & 4151.34 \\
\hline July & 24.450 & 16.300 & 12.225 & 307.07 & 7507.86 & 5005.24 & 3753.93 \\
\hline August & 20.062 & 12.003 & 9.002 & 295.24 & 5923.10 & 3543.77 & 2657.75 \\
\hline September & 21.559 & 14.373 & 10.779 & 302.20 & 6515.13 & 4343.52 & 3257.41 \\
\hline October & 21.106 & 14.071 & 10.553 & 301.38 & 6360.93 & 3035.20 & 3180.46 \\
\hline November & 21.237 & 14.158 & 10.618 & 301.62 & 6405.50 & 4270.34 & 3202.60 \\
\hline December & 14.423 & 9.615 & 7.211 & 286.66 & 4134.49 & 2756.24 & 2067.11 \\
\hline
\end{tabular}

Table 3.6: Rainfall Intensity (I), Rainfall Energy (E) and Rainfall Erosivity Factor (R) for Ohaji (Sands, Gravel and Clay)

\begin{tabular}{|c|c|c|c|c|c|c|c|}
\hline Months & $\begin{array}{c}\mathbf{I}_{\mathbf{3 0}} \\
(\mathrm{mm} / \mathrm{hr})\end{array}$ & $\begin{array}{c}\mathbf{I}_{45} \\
(\mathrm{~mm} / \mathrm{hr})\end{array}$ & $\begin{array}{c}\mathbf{I}_{60} \\
(\mathrm{~mm} / \mathrm{hr})\end{array}$ & $\begin{array}{c}\mathbf{E} \\
(\mathbf{M J m m} / \mathbf{h a})\end{array}$ & $\begin{array}{c}\mathrm{EI}_{30}(\mathbf{R}) \\
\left(\mathrm{MJmm} / \mathrm{hahr}^{-1}\right)\end{array}$ & $\begin{array}{c}\text { EI }_{45} \\
\left(\mathbf{M J m m} / \mathrm{hahr}^{-1}\right)\end{array}$ & $\begin{array}{c}\text { EI }_{60} \\
\left(\mathrm{MJmm} / \mathrm{hahr}^{-1}\right)\end{array}$ \\
\hline January & 8.899 & 5.933 & 4.449 & 267.99 & 2381.95 & 1590.01 & 1192.31 \\
\hline February & 25.338 & 16.892 & 12.669 & 308.44 & 7815.25 & 5210.17 & 3907.63 \\
\hline March & 24.797 & 16.531 & 12.398 & 307.61 & 7627.81 & 5085.10 & 3813.75 \\
\hline April & 20.289 & 13.527 & 10.145 & 299.86 & 6083.86 & 4056.21 & 3042.08 \\
\hline May & 24.255 & 16.165 & 12.124 & 366.74 & 7439.98 & 4958.45 & 3718.92 \\
\hline June & 29.474 & 19.953 & 14.737 & 314.29 & 9263.38 & 6271.03 & 4631.69 \\
\hline July & 20.923 & 13.949 & 10.462 & 301.05 & 6298.87 & 4199.35 & 3149.59 \\
\hline August & 19.490 & 12.993 & 9.745 & 298.30 & 5813.87 & 3875.81 & 2906.93 \\
\hline September & 20.729 & 13.819 & 10.365 & 300.69 & 6233.00 & 4155.24 & 3116.65 \\
\hline October & 22.597 & 13.731 & 10.298 & 300.44 & 6188.16 & 4125.34 & 3093.93 \\
\hline November & 22.581 & 15.054 & 11.290 & 303.99 & 6864.40 & 4576.27 & 3432.05 \\
\hline December & 19.231 & 12.821 & 9.615 & 297.78 & 5726.61 & 3817.84 & 2863.16 \\
\hline
\end{tabular}


International Journal of Engineering Applied Sciences and Technology, 2019

Vol. 4, Issue 6, ISSN No. 2455-2143, Pages 319-332

Published Online October 2019 in IJEAST (http://www.ijeast.com)

Table 3.7: Rainfall Intensity (I), Rainfall Energy (E) and Rainfall Erosivity Factor (R) for Okigwe (Sandstones, Limestones and Coal)

\begin{tabular}{|c|c|c|c|c|c|c|c|}
\hline Months & $\begin{array}{c}\mathbf{I}_{30} \\
(\mathrm{~mm} / \mathrm{hr})\end{array}$ & $\begin{array}{c}\mathbf{I}_{45} \\
(\mathrm{~mm} / \mathrm{hr})\end{array}$ & $\begin{array}{c}\text { I } 60 \\
(\mathrm{~mm} / \mathrm{hr})\end{array}$ & $\begin{array}{c}\mathbf{E} \\
(\mathbf{M J m m} / \mathbf{h a})\end{array}$ & $\begin{array}{c}\text { EI }_{30}(\mathbf{R}) \\
\left(\mathbf{M J m m} / \mathbf{h a h r}^{-1}\right)\end{array}$ & $\begin{array}{c}\text { EI }_{45} \\
\left(\mathrm{MJmm} / \mathrm{hahr}^{-1}\right)\end{array}$ & $\begin{array}{c}\text { EI }_{60} \\
\left(\mathrm{MJmm} / \mathrm{hahr}^{-1}\right)\end{array}$ \\
\hline January & 8.091 & 5.394 & 4.045 & 264.32 & 2138.61 & 1425.74 & 1069.17 \\
\hline February & 24.211 & 16.141 & 12.106 & 306.69 & 7425.27 & 4950.29 & 3712.79 \\
\hline March & 20.935 & 13.957 & 10.467 & 301.06 & 6302.69 & 4201.89 & 3151.20 \\
\hline April & 17.149 & 11.433 & 8.575 & 293.36 & 5030.83 & 3353.98 & 2515.56 \\
\hline May & 23.096 & 15.322 & 11.544 & 304.85 & 7040.82 & 4670.91 & 3519.19 \\
\hline June & 22.638 & 15.324 & 11.319 & 304.09 & 6883.99 & 4659.88 & 3441.99 \\
\hline July & 19.787 & 13.191 & 9.894 & 298.89 & 5914.14 & 3942.86 & 2957.22 \\
\hline August & 19.433 & 12.955 & 9.717 & 298.19 & 5794.73 & 3863.05 & 2897.51 \\
\hline September & 18.361 & 12.240 & 9.180 & 295.99 & 5434.67 & 3622.92 & 2717.19 \\
\hline October & 18.705 & 12.489 & 9.352 & 296.71 & 5549.96 & 3705.61 & 2774.83 \\
\hline November & 19.086 & 12.724 & 9.543 & 297.49 & 5677.89 & 3785.26 & 2838.95 \\
\hline December & 12.821 & 8.547 & 6.410 & 282.11 & 3616.93 & 2411.19 & 1808.33 \\
\hline
\end{tabular}

Table 3.8: Rainfall Intensity (I), Rainfall Energy (E) and Rainfall Erosivity Factor (R) for Orlu (Clayey Sands and Shales)

\begin{tabular}{|c|c|c|c|c|c|c|c|}
\hline Months & $\begin{array}{c}\mathbf{I}_{30} \\
(\mathbf{m m} / \mathbf{h r})\end{array}$ & $\begin{array}{c}\mathbf{I}_{45} \\
(\mathrm{~mm} / \mathrm{hr})\end{array}$ & $\begin{array}{c}\mathbf{I}_{60} \\
(\mathbf{m m} / \mathbf{h r})\end{array}$ & $\begin{array}{c}E \\
(\mathbf{M J m m} / \mathbf{h a})\end{array}$ & $\begin{array}{c}\mathbf{E I}_{30}(\mathbf{R}) \\
\left(\mathrm{MJmm} / \mathbf{h a h r}^{-1}\right)\end{array}$ & $\begin{array}{c}\mathbf{E I}_{45} \\
\left(\mathbf{M J m m} / \mathbf{h a h r}^{-1}\right)\end{array}$ & $\begin{array}{c}\text { EI }_{60} \\
\left(\mathbf{M J m m} / h^{-1}\right)\end{array}$ \\
\hline January & 8.899 & 5.933 & 4.449 & 267.99 & 2381.95 & 1590.01 & 1192.31 \\
\hline February & 20.270 & 13.514 & 10.135 & 299.82 & 6077.35 & 4051.77 & 3038.68 \\
\hline March & 19.309 & 12.873 & 6.654 & 297.94 & 5752.92 & 3835.38 & 2876.31 \\
\hline April & 18.237 & 12.157 & 9.118 & 295.73 & 5393.23 & 3595.19 & 2696.47 \\
\hline May & 22.351 & 14.896 & 11.170 & 303.58 & 6785.32 & 4522.13 & 3391.29 \\
\hline June & 21.726 & 14.707 & 10.863 & 302.49 & 6571.89 & 448.72 & 3285.95 \\
\hline July & 20.146 & 13.431 & 10.073 & 299.58 & 6035.34 & 4023.66 & 3017.67 \\
\hline August & 17.547 & 11.698 & 8.773 & 294.24 & 5163.03 & 3442.02 & 2581.37 \\
\hline September & 19.427 & 12.951 & 9.713 & 298.17 & 5792.63 & 3861.65 & 2896.16 \\
\hline October & 18.559 & 12.373 & 9.279 & 296.41 & 5501.07 & 3667.48 & 2750.39 \\
\hline November & 17.473 & 11.649 & 8.737 & 294.08 & 5138.46 & 3425.74 & 2569.38 \\
\hline December & 12.821 & 8.547 & 6.410 & 282.11 & 3616.93 & 2411.19 & 1808.33 \\
\hline
\end{tabular}


International Journal of Engineering Applied Sciences and Technology, 2019

Vol. 4, Issue 6, ISSN No. 2455-2143, Pages 319-332

Published Online October 2019 in IJEAST (http://www.ijeast.com)

Table 3.9: Rainfall Intensity (I), Rainfall Energy (E) and Rainfall Erosivity Factor (R) for Owerri West (Sands and Clay)

\begin{tabular}{|c|c|c|c|c|c|c|c|}
\hline Months & $\begin{array}{c}\mathbf{I}_{30} \\
(\mathbf{m m} / \mathbf{h r})\end{array}$ & $\begin{array}{c}\mathbf{I}_{45} \\
(\mathrm{~mm} / \mathrm{hr})\end{array}$ & $\begin{array}{c}\text { I } 60 \\
(\mathbf{m m} / \mathbf{h r})\end{array}$ & $\begin{array}{c}\mathbf{E} \\
(\mathbf{M J m m} / \mathbf{h a})\end{array}$ & $\begin{array}{c}\mathbf{E I}_{30}(\mathbf{R}) \\
\left(\mathbf{M J m m} / \mathbf{h a h r}^{-1}\right)\end{array}$ & $\begin{array}{c}\mathbf{E I}_{45} \\
\left(\mathrm{MJmm} / \mathrm{hahr}^{-1}\right)\end{array}$ & $\begin{array}{c}\text { EI }_{60} \\
\left(\mathrm{MJmm} / \mathrm{hahr}^{-1}\right)\end{array}$ \\
\hline January & 6.525 & 4.315 & 3.236 & 255.69 & 1668.38 & 1103.30 & 829.41 \\
\hline February & 16.891 & 11.261 & 8.446 & 292.77 & 4945.18 & 3296.88 & 2472.74 \\
\hline March & 22.154 & 14.769 & 11.077 & 303.25 & 6718.20 & 4478.69 & 3359.10 \\
\hline April & 18.841 & 12.560 & 9.420 & 296.99 & 5595.59 & 3730.19 & 2797.65 \\
\hline May & 22.103 & 14.730 & 11.048 & 303.15 & 6700.52 & 4465.40 & 3349.20 \\
\hline June & 30.918 & 20.929 & 15.489 & 316.21 & 9776.58 & 6617.96 & 4897.78 \\
\hline July & 18.113 & 12.076 & 9.057 & 295.47 & 5351.85 & 3568.09 & 2404.36 \\
\hline August & 22.805 & 15.204 & 11.403 & 304.37 & 6941.16 & 4627.64 & 3470.73 \\
\hline September & 16.347 & 10.898 & 8.173 & 291.50 & 4765.15 & 3176.77 & 2382.43 \\
\hline October & 20.306 & 13.537 & 10.153 & 299.89 & 6089.57 & 4059.61 & 3044.78 \\
\hline November & 18.279 & 12.186 & 9.139 & 295.82 & 5407.29 & 3604.86 & 2703.49 \\
\hline December & 6.571 & 4.380 & 3.285 & 256.27 & 1683.95 & 1122.46 & 841.85 \\
\hline
\end{tabular}

Table 3.10: Mean Rainfall Erosivity Factor $(\mathbf{R})$ for all the Locations

\begin{tabular}{|l|c|}
\hline \multicolumn{1}{|c|}{ Soil Texture/Location } & $\begin{array}{c}\text { Mean Rainfall } \\
\text { Erosivity Factor (R) } \\
\text { (MJmm/hahr-1) }\end{array}$ \\
\hline $\begin{array}{l}\text { Clay and Shales (Ehime } \\
\text { Mbano) }\end{array}$ & 6054.93 \\
\hline $\begin{array}{l}\text { Sandstones (Ideato } \\
\text { North) }\end{array}$ & 5211.48 \\
\hline $\begin{array}{l}\text { Clay, sandstones, lignite } \\
\text { and Shale(Ikeduru) }\end{array}$ & 5977.29 \\
\hline $\begin{array}{l}\text { Sands, Clay and Swamps } \\
\text { (Oguta) }\end{array}$ & 6171.57 \\
\hline $\begin{array}{l}\text { Sand, Gravel and Clay } \\
\text { (OhajiEgbema) }\end{array}$ & 6478.06 \\
\hline $\begin{array}{l}\text { Sandstones, Limestones } \\
\text { and Coal (Okigwe) }\end{array}$ & 5567.54 \\
\hline $\begin{array}{l}\text { Clayey sands and Shale } \\
\text { (Orlu) }\end{array}$ & 5350.84 \\
\hline $\begin{array}{l}\text { Sands and Clay (Owerri } \\
\text { West })\end{array}$ & 5470.29 \\
\hline
\end{tabular}

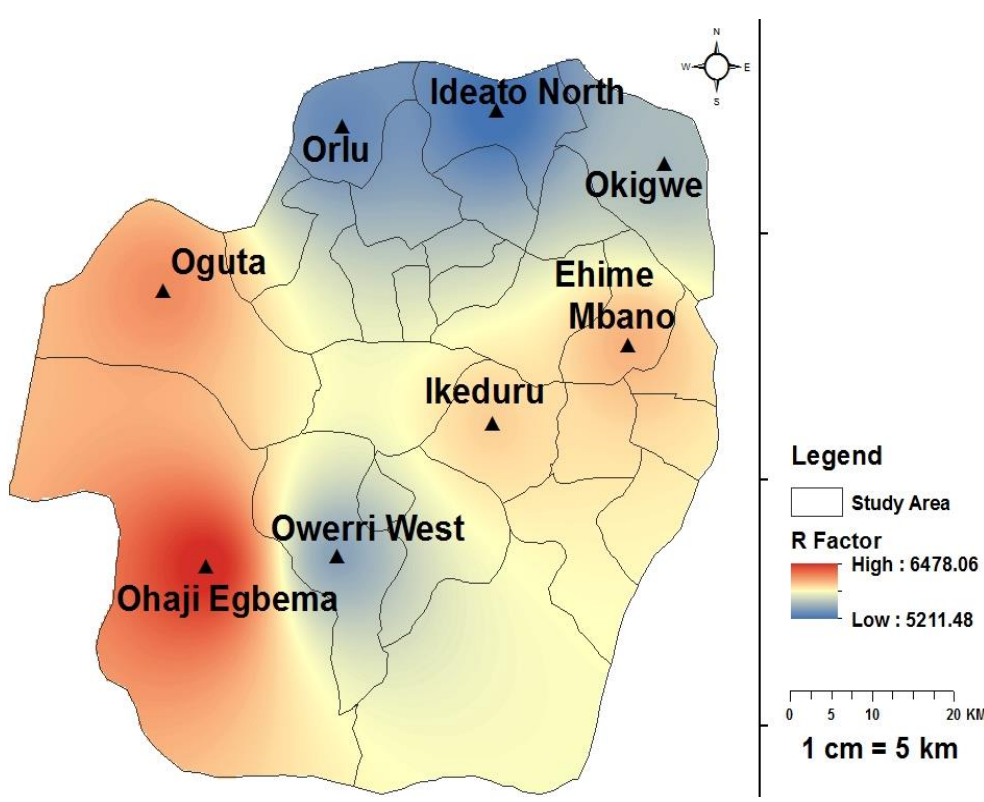

Figure 2: Rainfall Erosivity Map of Imo State.

Table 3.11 contains classification information on the corresponding rainfall erosivity range values for various rainfall erosivity classes and from the range of values obtained for each of the locations it was observed that all the locations within Imo State fall within the erosivity class of medium-strong erosivity on a range of $5211.48-6478.06 \mathrm{MJmmha}^{-1} \mathrm{hr}^{-1}$. 
Table 3.11: Classification of Rainfall Erosivity Factor $(\mathbf{R})$

\begin{tabular}{|l|l|}
\hline \multicolumn{1}{|c|}{$\begin{array}{c}\text { EROSIVITY } \\
\left(\mathbf{M J m m h a}^{-1} \mathbf{h r}^{-\mathbf{1}}\right)\end{array}$} & \multicolumn{1}{c|}{$\begin{array}{c}\text { EROSIVITY } \\
\text { CLASS }\end{array}$} \\
\hline $\mathrm{R} \leq 2452$ & low erosivity \\
\hline $2452<\mathrm{R} \leq 4905$ & Medium erosivity \\
\hline $4905<\mathrm{R} \leq 7357$ & medium- strong erosivity \\
\hline $7357<\mathrm{R} \leq 9810$ & strong erosivity \\
\hline$<9810$ & very strong erosivity \\
\hline
\end{tabular}

(Source: Carvalho, (2008); Oliviera et al., 2012)

\section{1: Correlation Analysis of Data}

The rainfall amount, rainfall intensity (I), rainfall energy (E) and rainfall erosivity factor $(\mathrm{R})$ for the different rainfall intensities for each of the locations (different soil groups) were subjected to correlation analysis to obtain the coefficient of correlation between the parameters. Tables 3.12-3.20 presents the values of the coefficient of correlation for each parameters and the location

Table 3.12: Correlation of Mean Rainfall Amount and Rainfall Days for all the Locations (1986- 2016)

\begin{tabular}{|l|c|c|c|c|c|c|c|c|}
\hline & Ehime.Mbano & Ideato.North & Ikeduru & Oguta & Ohaji & Okigwe & Orlu & Owerri \\
\hline Ehime.Mbano & 1 & & & & & & & \\
\hline Ideato.North & $.997^{* *}$ & 1 & & & & & & \\
\hline Ikeduru & $.994^{* *}$ & $.989^{* *}$ & 1 & & & & & \\
\hline Oguta & $.992^{* *}$ & $.988^{* *}$ & $.983^{* *}$ & 1 & & & & \\
\hline Ohaji & $.992^{* *}$ & $.992^{* *}$ & $.995^{* *}$ & $.987^{* *}$ & 1 & & & \\
\hline Okigwe & $.998^{* *}$ & $.997^{* *}$ & $.993^{* *}$ & $.985^{* *}$ & $.988^{* *}$ & 1 & & \\
\hline Orlu & $.996^{* *}$ & $.997^{* *}$ & $.985^{* *}$ & $.993^{* *}$ & $.987^{* *}$ & $.995^{* *}$ & 1 & \\
\hline Owerri & $.964^{* *}$ & $.960^{* *}$ & $.986^{* *}$ & $.941^{* *}$ & $.977^{* *}$ & $.967^{* *}$ & $.948^{* *}$ & 1 \\
\hline **. Correlation is significant at the 0.05 level (2-tailed) \\
\end{tabular}

Table 3.12 above shows the correlation of mean rainfall amount for the various locations considered in this study from 19862016. From the result it is observed that Ehime Mbano, Ideato North, Ikeduru, Oguta, Ohajiegbema, Okigwe, Orlu and Owerri

Table 3.13: Correlation of Rainfall Intensity (I), Rainfall Energy (E) and Rainfall Erosivity Factor (R) for Ehime Mbano (Clay and Shales)

\begin{tabular}{|c|c|c|c|c|c|c|c|}
\hline & $\begin{array}{c}\mathbf{I}_{30} \\
(\mathbf{m m} / \mathbf{h r})\end{array}$ & $\begin{array}{c}\mathbf{I}_{45} \\
(\mathbf{m m} / \mathbf{h r})\end{array}$ & $\begin{array}{c}\mathbf{I}_{60} \\
(\mathrm{~mm} / \mathrm{hr})\end{array}$ & $\begin{array}{c}E \\
(\mathbf{M J m m} / \mathbf{h a})\end{array}$ & $\mathbf{E I}_{30}(\mathbf{R})$ & $\mathbf{E I}_{45}(\mathbf{R})$ & $\mathbf{E I}_{60}(\mathbf{R})$ \\
\hline $\mathbf{I}_{30}(\mathbf{m m} / \mathbf{h r})$ & 1 & & & & & & \\
\hline $\mathbf{I}_{45}(\mathrm{~mm} / \mathrm{hr})$ & $1.000^{* *}$ & 1 & & & & & \\
\hline $\mathrm{I}_{60}(\mathrm{~mm} / \mathrm{hr})$ & $1.000^{* *}$ & $1.000^{* * *}$ & 1 & & & & \\
\hline E (MJmm/ha) & $.976^{* *}$ & $.975^{* *}$ & $.976^{* *}$ & 1 & & & \\
\hline $\mathbf{E I}_{30}(\mathbf{R})$ & $1.000^{* *}$ & $.999^{* *}$ & $1.000^{* *}$ & $.970^{* *}$ & 1 & & \\
\hline $\mathbf{E I}_{45}(\mathbf{R})$ & $.999^{* *}$ & $1.000^{* * *}$ & $.999^{* *}$ & $.969^{* *}$ & $1.000^{* * *}$ & 1 & \\
\hline $\mathbf{E I}_{60}(\mathbf{R})$ & $1.000^{* *}$ & $.999^{* *}$ & $1.000^{* *}$ & $.970^{* * *}$ & $1.000^{* * *}$ & $1.000^{* *}$ & 1 \\
\hline
\end{tabular}

Table 3.13 above shows the correlation of rainfall intensity (I), rainfall energy $(\mathrm{E})$ and rainfall erosivity factor $(\mathrm{R})$ for Ehime Mbano (Clay and Shales). From the result it is observed that rainfall intensity (I), rainfall energy (E) and rainfall erosivity all show a strong/perfect correlation with the others location within the regions since all had a correlation coefficient of above 0.9 and the results were all statistically significant at $5 \%$.

Table 3.14: Correlation on Rainfall Intensity (I), Rainfall Energy (E) and Rainfall Erosivity Factor (R) for Ideato North (Sandstones)

\begin{tabular}{|l|c|c|c|c|c|c|c|}
\hline & $\begin{array}{c}\mathbf{I}_{30} \\
(\mathbf{m m} / \mathbf{h r})\end{array}$ & $\begin{array}{c}\mathbf{I}_{45} \\
(\mathbf{m m} / \mathbf{h r})\end{array}$ & $\begin{array}{c}\mathbf{I}_{60} \\
(\mathbf{m m} / \mathbf{h r})\end{array}$ & $\begin{array}{c}\mathbf{E} \\
(\mathbf{M J m m} / \mathbf{h a})\end{array}$ & $\mathbf{E I}_{30}(\mathbf{R})$ & $\mathbf{E I}_{45}(\mathbf{R})$ & $\mathbf{E I}_{60}(\mathbf{R})$ \\
\hline $\mathbf{I}_{\mathbf{3 0}}(\mathbf{m m} / \mathbf{h r})$ & 1 & & & & & & \\
\hline $\mathbf{I}_{45}(\mathbf{m m} / \mathbf{h r})$ & $1.000^{* * *}$ & 1 & & & & & \\
\hline
\end{tabular}


International Journal of Engineering Applied Sciences and Technology, 2019

Vol. 4, Issue 6, ISSN No. 2455-2143, Pages 319-332

Published Online October 2019 in IJEAST (http://www.ijeast.com)

\begin{tabular}{|l|c|c|c|c|c|c|c|}
\hline $\mathbf{I}_{60}(\mathbf{m m} / \mathbf{h r})$ & $1.000^{* *}$ & $1.000^{* *}$ & 1 & & & & \\
\hline $\mathbf{E}(\mathbf{M J m m} / \mathbf{h a})$ & $.986^{* *}$ & $.984^{* *}$ & $.986^{* *}$ & 1 & & & \\
\hline $\mathbf{E I}_{\mathbf{3 0}}(\mathbf{R})$ & $1.000^{* *}$ & $1.000^{* *}$ & $1.000^{* *}$ & $.982^{* *}$ & 1 & & \\
\hline $\mathbf{E I}_{45} \mathbf{( R )}$ & $.956^{* *}$ & $.957^{* *}$ & $.956^{* *}$ & $.929^{* *}$ & $.957^{* *}$ & 1 & \\
\hline $\mathbf{E I}_{60}(\mathbf{R})$ & $1.000^{* *}$ & $1.000^{* *}$ & $1.000^{* *}$ & $.982^{* *}$ & $1.000^{* *}$ & $.957^{* *}$ & 1 \\
\hline
\end{tabular}

Table 3.14 above shows the correlation of rainfall intensity (I), rainfall energy (E) and rainfall erosivity factor (R) for Ideato North (Sandstones). From the result it was observed that rainfall intensity (I), rainfall energy $(E)$ and rainfall erosivity factor $(R)$ for Ideato North (Sandstone) all show a strong/perfect correlation with the others at Ideato North since all had a correlation coefficient of above 0.95 which is about $95 \%$ strength and the results were all statistically significant at $5 \%$.

Table 3.15: Correlation on Rainfall Intensity (I), Rainfall Energy (E) and Rainfall Erosivity Factor (R) for Ikeduru (Clays, Sandstones, Lignite and Shales)

\begin{tabular}{|l|l|l|l|l|l|l|l|}
\hline & \multicolumn{1}{|c|}{$\begin{array}{c}\mathbf{I}_{\mathbf{3 0}} \\
(\mathbf{m m} / \mathbf{h r})\end{array}$} & $\begin{array}{c}\mathbf{I}_{45} \\
(\mathbf{m m} / \mathbf{h r})\end{array}$ & $\begin{array}{c}\mathbf{I}_{\mathbf{6 0}} \\
(\mathbf{m m} / \mathbf{h r})\end{array}$ & $\begin{array}{c}\mathbf{E} \\
(\mathbf{M J m m} / \mathbf{h a})\end{array}$ & $\mathbf{E I}_{\mathbf{3 0}}(\mathbf{R})$ & $\mathbf{E I}_{\mathbf{4 5}}(\mathbf{R})$ & $\mathbf{E I}_{\mathbf{6 0}}(\mathbf{R})$ \\
\hline $\mathbf{I}_{\mathbf{3 0}}(\mathbf{m m} / \mathbf{h r})$ & 1 & & & & & & \\
\hline $\mathbf{I}_{\mathbf{4 5}}(\mathbf{m m} / \mathbf{h r})$ & $1.000^{* *}$ & 1 & & & & & \\
\hline $\mathbf{I}_{\mathbf{6 0}}(\mathbf{m m} / \mathbf{h r})$ & $1.000^{* *}$ & $1.000^{* *}$ & 1 & & & & \\
\hline $\mathbf{E}(\mathbf{M J m m} / \mathbf{h a})$ & $.978^{* *}$ & $.974^{* *}$ & $.978^{* *}$ & 1 & & & \\
\hline $\mathbf{E I}_{\mathbf{3 0}}(\mathbf{R})$ & $.999^{* *}$ & $1.000^{* *}$ & $.999^{* *}$ & $.972^{* *}$ & 1 & & \\
\hline $\mathbf{E I}_{45}(\mathbf{R})$ & $.999^{* *}$ & $1.000^{* *}$ & $.999^{* *}$ & $.968^{* *}$ & $.999^{* *}$ & 1 & \\
\hline $\mathbf{E I}_{\mathbf{6 0}}(\mathbf{R})$ & $1.000^{* *}$ & $1.000^{* *}$ & $1.000^{* *}$ & $.972^{* *}$ & $1.000^{* *}$ & $1.000^{* *}$ & 1 \\
\hline
\end{tabular}

Table 3.15 above shows the correlation of rainfall intensity (I), rainfall energy (E) and rainfall erosivity factor (R) for Ikeduru (Clays, Sandstones, Lignite and shales). From the result it was observed that rainfall intensity (I), rainfall energy (E) and rainfall erosivity factor (R) for Ikeduru (Clays, Sandstones, Lignite and

Table 3.16: Correlation on Rainfall Intensity (I), Rainfall Energy (E) and Rainfall Erosivity Factor (R) for Oguta (Sands, Clays and Swamps)

\begin{tabular}{|l|c|c|c|c|c|c|c|}
\hline & $\begin{array}{c}\mathbf{I}_{\mathbf{3 0}} \\
(\mathbf{m m} / \mathbf{h r})\end{array}$ & $\begin{array}{c}\mathbf{I}_{\mathbf{4 5}} \\
(\mathbf{m m} / \mathbf{h r})\end{array}$ & $\begin{array}{c}\mathbf{I}_{60} \\
(\mathbf{m m} / \mathbf{h r})\end{array}$ & $\begin{array}{c}\mathbf{E} \\
(\mathbf{M J m m} / \mathbf{h a})\end{array}$ & $\mathbf{E I}_{\mathbf{3 0}}(\mathbf{R})$ & $\mathbf{E I}_{\mathbf{4 5}}(\mathbf{R})$ & $\mathbf{E I}_{\mathbf{6 0}}(\mathbf{R})$ \\
\hline $\mathbf{I}_{\mathbf{3 0}}(\mathbf{m m} / \mathbf{h r})$ & 1 & & & & & & \\
\hline $\mathbf{I}_{\mathbf{4 5}}(\mathbf{m m} / \mathbf{h r})$ & $.991^{* * *}$ & 1 & & & & & \\
\hline $\mathbf{I}_{\mathbf{6 0}}(\mathbf{m m} / \mathbf{h r})$ & $.991^{* *}$ & $1.000^{* *}$ & 1 & & & & \\
\hline $\mathbf{E}(\mathbf{M J m m} / \mathbf{h a})$ & $.987^{* *}$ & $.987^{* *}$ & $.990^{* * *}$ & 1 & & & \\
\hline $\mathbf{E I}_{\mathbf{3 0}}(\mathbf{R})$ & $1.000^{* *}$ & $.993^{* *}$ & $.993^{* *}$ & $.986^{* *}$ & 1 & & \\
\hline $\mathbf{E I}_{45}(\mathbf{R})$ & $.935^{* *}$ & $.942^{* *}$ & $.940^{* *}$ & $.918^{* *}$ & $.939^{* *}$ & 1 & \\
\hline $\mathbf{E I}_{\mathbf{6 0}}(\mathbf{R})$ & $.989^{* *}$ & $.999^{* *}$ & $.999^{* * *}$ & $.984^{* *}$ & $.992^{* *}$ & $.942^{* *}$ & 1 \\
\hline
\end{tabular}

Table 3.16 above shows the correlation of rainfall intensity (I), rainfall energy (E) and rainfall erosivity factor (R) for Oguta (Sands, Clays and Swamps). From the result it is observed that rainfall intensity (I), rainfall energy (E) and rainfall erosivity shales) all show a strong/perfect correlation with the others at Ikeduru since all had a correlation coefficient of above 0.95 which is about 95\% strength and the results were all statistically significant at $5 \%$.

Table 3.17: Correlation on Rainfall Intensity (I), Rainfall Energy (E) and Rainfall Erosivity Factor (R) for Ohajiegbema (Sands, Gravel and Clay)

\begin{tabular}{|c|c|c|c|c|c|c|c|}
\hline & $\begin{array}{c}\mathbf{I}_{30} \\
(\mathbf{m m} / \mathbf{h r})\end{array}$ & $\begin{array}{c}\mathbf{I}_{45} \\
(\mathbf{m m} / \mathbf{h r})\end{array}$ & $\begin{array}{c}\mathbf{I}_{60} \\
(\mathbf{m m} / \mathbf{h r})\end{array}$ & $\begin{array}{c}\mathbf{E} \\
(\mathbf{M J m m} / \mathbf{h a})\end{array}$ & $\mathbf{E I}_{30}(\mathbf{R})$ & $\mathbf{E I}_{45}(\mathbf{R})$ & $\mathbf{E I} 60(\mathbf{R})$ \\
\hline
\end{tabular}


International Journal of Engineering Applied Sciences and Technology, 2019

Vol. 4, Issue 6, ISSN No. 2455-2143, Pages 319-332

Published Online October 2019 in IJEAST (http://www.ijeast.com)

\begin{tabular}{|l|c|c|c|c|c|c|c|}
\hline $\mathbf{I}_{\mathbf{3 0}}(\mathbf{m m} / \mathbf{h r})$ & 1 & & & & & \\
\hline $\mathbf{I}_{45}(\mathbf{m m} / \mathbf{h r})$ & $.993^{* *}$ & 1 & & & & & \\
\hline $\mathbf{I}_{\mathbf{6 0}}(\mathbf{m m} / \mathbf{h r})$ & $.993^{* *}$ & $1.000^{* *}$ & 1 & & & & \\
\hline $\mathbf{E}(\mathbf{M J m m} / \mathbf{h a})$ & $.633^{*}$ & $.636^{*}$ & $.642^{*}$ & 1 & & & \\
\hline $\mathbf{E I}_{\mathbf{3 0}}(\mathbf{R})$ & $.992^{* *}$ & $1.000^{* *}$ & $1.000^{* *}$ & $.639^{*}$ & 1 & & \\
\hline $\mathbf{E I}_{\mathbf{4} 5}(\mathbf{R})$ & $.992^{* *}$ & $1.000^{* *}$ & $.999^{* *}$ & $.634^{*}$ & $1.000^{* *}$ & 1 & \\
\hline EI60 $(\mathbf{R})$ & $.992^{* *}$ & $1.000^{* *}$ & $1.000^{* *}$ & $.639^{*}$ & $1.000^{* *}$ & $1.000^{* *}$ & 1 \\
\hline
\end{tabular}

Table 3.17 above shows the correlation of rainfall intensity (I), rainfall energy (E) and rainfall erosivity factor (R) for Ohajiegbema (Sands, Gravel and Clays). It was observed that rainfall intensity (I) and rainfall erosivity factor (R) for Ohajiegbema (Sands, Gravel and Clays) all showed a strong/perfect correlation of above 0.95 which is $95 \%$ strength while rainfall energy (E) showed a fairly strong correlation of about 0.63 with the others at Ohajiegbema, but all the results were statistically significant at $5 \%$.

Table 3.18: Correlation on Rainfall Intensity (I), Rainfall Energy (E) and Rainfall Erosivity Factor (R) for Okigwe (Sandstones, Limestones and Coal)

\begin{tabular}{|c|c|c|c|c|c|c|c|}
\hline & $\begin{array}{c}\mathbf{I}_{30} \\
(\mathbf{m m} / \mathbf{h r})\end{array}$ & $\begin{array}{c}\mathbf{I}_{45} \\
(\mathbf{m m} / \mathbf{h r})\end{array}$ & $\begin{array}{c}\mathbf{I}_{\mathbf{6 0}} \\
(\mathbf{m m} / \mathbf{h r})\end{array}$ & $\begin{array}{c}\mathbf{E} \\
(\mathbf{M J m m} / \mathbf{h a})\end{array}$ & $\mathbf{E I}_{30}(\mathbf{R})$ & $\mathbf{E I}_{45}(\mathbf{R})$ & EI $_{60}(\mathbf{R})$ \\
\hline $\mathbf{I}_{30}(\mathbf{m m} / \mathbf{h r})$ & 1 & & & & & & \\
\hline $\mathbf{I}_{45}(\mathrm{~mm} / \mathrm{hr})$ & $1.000^{* *}$ & 1 & & & & & \\
\hline$I_{60}(\mathrm{~mm} / \mathrm{hr})$ & $1.000^{* *}$ & $1.000^{* *}$ & 1 & & & & \\
\hline E (MJmm/ha) & $.986^{* *}$ & $.985^{* * *}$ & $.986^{* *}$ & 1 & & & \\
\hline $\mathbf{E I}_{30}(\mathbf{R})$ & $1.000^{* *}$ & $1.000^{* *}$ & $1.000^{* *}$ & $.983^{* *}$ & 1 & & \\
\hline EI45 & $1.000^{* * *}$ & $1.000^{* * *}$ & $1.000^{* *}$ & $.982^{* *}$ & $1.000^{* *}$ & 1 & \\
\hline EI $_{60}$ & $1.000^{* * *}$ & $1.000^{* *}$ & $1.000^{* *}$ & $.983^{* *}$ & $1.000^{* *}$ & $1.000^{* *}$ & 1 \\
\hline
\end{tabular}

Table 3.18 above shows the correlation of rainfall intensity (I), rainfall energy (E) and rainfall erosivity factor (R) for Okigwe (Sandstones, Limestones and Coal). From the result it was observed that rainfall intensity (I), rainfall energy (E) and rainfall erosivity factor (R) for Okigwe (Sandstones, Limestones and
Coal) all showed a strong/perfect correlation with the others at Okigwe since all had a correlation coefficient of above 0.95 which is about $95 \%$ strength and the results were all statistically significant at $5 \%$.

Table 3.19: Correlation on Rainfall Intensity (I), Rainfall Energy (E) and Rainfall Erosivity Factor (R) for Orlu (Clayey Sands and Shales)

\begin{tabular}{|l|c|c|c|c|c|c|c|}
\hline & $\begin{array}{c}\mathbf{I}_{30} \\
(\mathbf{m m} / \mathbf{h r})\end{array}$ & $\begin{array}{c}\mathbf{I}_{\mathbf{4 5}} \\
(\mathbf{m m} / \mathbf{h r})\end{array}$ & $\begin{array}{c}\mathbf{I}_{\mathbf{6 0}} \\
(\mathbf{m m} / \mathbf{h r})\end{array}$ & $\begin{array}{c}\mathbf{E} \\
(\mathbf{M J m m} / \mathbf{h a})\end{array}$ & $\mathbf{E I}_{\mathbf{3 0}}(\mathbf{R})$ & $\mathbf{E I}_{\mathbf{4 5}}(\mathbf{R})$ & $\mathbf{E I}_{\mathbf{6 0}}(\mathbf{R})$ \\
\hline $\mathbf{I}_{\mathbf{3 0}}(\mathbf{m m} / \mathbf{h r})$ & 1 & & & & & & \\
\hline $\mathbf{I}_{\mathbf{4 5}}(\mathbf{m m} / \mathbf{h r})$ & $1.000^{* *}$ & 1 & & & & & \\
\hline $\mathbf{I}_{\mathbf{6 0}}(\mathbf{m m} / \mathbf{h r})$ & $.902^{* *}$ & $.903^{* *}$ & 1 & & & & \\
\hline $\mathbf{E}(\mathbf{M J m m} / \mathbf{h a})$ & $.991^{* *}$ & $.990^{* *}$ & $.889^{* *}$ & 1 & & & \\
\hline $\mathbf{E I}_{\mathbf{3 0}}(\mathbf{R})$ & $1.000^{* *}$ & $1.000^{* *}$ & $.902^{* *}$ & $.989^{* *}$ & 1 & & \\
\hline $\mathbf{E I}_{\mathbf{4 5}}(\mathbf{R})$ & .405 & .383 & .314 & .444 & .400 & 1 & \\
\hline $\mathbf{E I}_{\mathbf{6 0}}(\mathbf{R})$ & $1.000^{* *}$ & $1.000^{* *}$ & $.902^{* *}$ & $.989^{* *}$ & $1.000^{* *}$ & .400 & 1 \\
\hline
\end{tabular}




\section{International Journal of Engineering Applied Sciences and Technology, 2019 \\ Vol. 4, Issue 6, ISSN No. 2455-2143, Pages 319-332 \\ Published Online October 2019 in IJEAST (http://www.ijeast.com)}

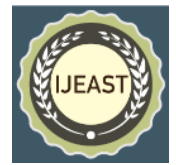

Table 3.19 above shows the correlation of rainfall intensity (I), rainfall energy (E) and rainfall erosivity factor (R) for Orlu (Clayey, Sands, and Shales). From the result it is observed that rainfall intensity (I), rainfall energy (E), $\mathrm{EI}_{30}$ and $\mathrm{EI}_{60}$ for Orlu (Clayey, Sands, and Shales) all showed a strong/perfect

Table 3.20: Correlation on Rainfall Intensity (I), Rainfall Energy (E) and Rainfall Erosivity Factor (R) for Owerri West (Sands and Clay)

\begin{tabular}{|c|c|c|c|c|c|c|c|}
\hline & $\begin{array}{c}\mathbf{I}_{\mathbf{3 0}} \\
(\mathrm{mm} / \mathrm{hr})\end{array}$ & $\begin{array}{c}\mathrm{I}_{45} \\
(\mathrm{~mm} / \mathrm{hr})\end{array}$ & $\begin{array}{c}\mathbf{I}_{60} \\
(\mathrm{~mm} / \mathrm{hr})\end{array}$ & $\begin{array}{c}\mathrm{E} \\
\text { (MJmm/ha) }\end{array}$ & $\mathbf{E I}_{30}(\mathbf{R})$ & $\mathbf{E I}_{45}(\mathbf{R})$ & $\mathrm{EI}_{60}(\mathbf{R})$ \\
\hline I30 (mm/hr) & 1 & & & & & & \\
\hline $\mathrm{I}_{45}(\mathrm{~mm} / \mathrm{hr})$ & $1.000^{* *}$ & 1 & & & & & \\
\hline $\mathrm{I}_{60}(\mathrm{~mm} / \mathrm{hr})$ & $1.000^{* *}$ & $1.000^{* *}$ & 1 & & & & \\
\hline E (MJmm/ha) & $.969^{* *}$ & $.966^{* *}$ & $.969^{* *}$ & 1 & & & \\
\hline $\mathrm{EI}_{30}(\mathbf{R})$ & $1.000^{* *}$ & $1.000^{* *}$ & $1.000^{* *}$ & $.961^{* *}$ & 1 & & \\
\hline $\mathbf{E I}_{45}$ & $.999^{* *}$ & $1.000^{* *}$ & $.999^{* *}$ & $.958^{* *}$ & $1.000^{* *}$ & 1 & \\
\hline $\mathbf{E I}_{60}$ & $.996^{* *}$ & $.997^{* *}$ & $.996^{* *}$ & $.954^{* *}$ & $.998^{* *}$ & $.998^{* *}$ & 1 \\
\hline
\end{tabular}

Table 3.20 above shows the correlation of rainfall intensity (I), rainfall energy (E) and rainfall erosivity factor $(\mathrm{R})$ for Owerri (Sands and Clay). It was observed from the result that rainfall intensity (I), rainfall energy (E) and rainfall erosivity factor (R) for Owerri (Sands and Clay) all showed a strong/perfect correlation with the others at Owerri since all had a correlation coefficient of above 0.95 which is about $95 \%$ strength and the results were all statistically significant at 5\%. Furthermore an ANOVA was carried out on rainfall erosivity factor for all the locations and it was observed that they did not show any significance since p-value > 0.05 , which also signifies no variation in rainfall properties since all the locations considered fall within the same region.

\section{DISCUSSIONS}

From the values displayed from Tables 3.1-3.10, it was observed that as rainfall duration increased the ratio of rainfall amount to duration (rainfall intensity) decreased accordingly. Likewise the rainfall erosivity factor $(\mathrm{R})$ at different rainfall intensities also decreased with increase in rainfall duration. This relationship is dependent on the fact that as rainfall duration increases the soil undergoes saturation and pore spaces are filled thus exceeding infiltration capacity at this point the soil particles no longer undergo detachment or splash but movement so therefore as rainfall duration increases rainfall intensity keeps decreasing on consistent distribution (Ojha and Michael, 2003). According to Salako, (2010) and Salako (2006) rainfall erosivity factor $(\mathrm{R})$ decreased with increase in rainfall duration, and it further stated that rainfall erosivity $(\mathrm{R})$ at 15 minutes rainfall intensity gives a better and more precise range of values for erosion prediction and management when compared to higher intensities. For all the locations the rainfall erosivity factor $(\mathrm{R})$ for each of the months varied between 1668.38 9776.58 $\mathrm{MJmmha}^{-1} \mathrm{hr}^{-1}$, the highest rainfall erosivity values were observed in June (rainy season) and the lowest rainfall correlation of about 0.90 which is about $90 \%$ strength with others while $\mathrm{EI}_{45}$ showed a very poor correlation of about 0.40 with the rainfall intensity (I), rainfall energy (E) and rainfall erosivity factor (R) in Orlu. 


\section{International Journal of Engineering Applied Sciences and Technology, 2019 \\ Vol. 4, Issue 6, ISSN No. 2455-2143, Pages 319-332 \\ Published Online October 2019 in IJEAST (http://www.ijeast.com)}

The mean annual rainfall erosivity $(\mathrm{R})$ for all the soil texture groups/locations was found to be between 5211.48 - 6478.06 $\mathrm{MJmmha}^{-1} \mathrm{hr}^{-1}$.

The study area falls within the range of medium to high rainfall erosivity with a value range of 5211.48-6478.06 $\mathrm{MJmmha}^{-1} \mathrm{hr}^{-}$ 1.

Coefficient of correlation for all the soil group zones/locations considered is 0.95 and above except for Orlu and Ohajiegbema which showed correlation coefficients of 0.40 and 0.60 at $\mathrm{EI}_{45}$ and $\mathrm{E}$ respectively.

ANOVA showed that al the locations were statistically significant at $5 \%$ thus reflecting uniform/similar rainfall characteristics

\section{ACKNOWLEDGEMENT}

The authors would like to acknowledge TETFUND (Tertiary Education Trust Fund) for sponsoring this study as well acknowledge NIMET (Nigerian Metrological Agency) for providing the rainfall data that was used to carry out this research.

\section{REFERENCES}

[1] Akinbile, C.O., Erazua, A.E., Babalola, T.E. and F.O.Ajibade (2016) Environmental Implications of Animal wastes pollution on Agricultural soil and water quality. Soil and Water Research 11 (3): 229-250.

[2] Bai, Z.G., Dent, D.L., Olsson, L and M.E. Schaepman (2008), Global Assessment of land degradation and Improvement, Identification by remote sensing. Report 2008/01, ISRIC, Wageningen 26-36 pp.

[3] Balogun, I., Adegun, O. and O. Adeaga (2012) an assessment of Rainfall Erosivity in parts of eastern Nigeria. A case study of Owerri and Enugu. Hydrology of Disaster Management 3: 259269

[4] Carvalho, N.O., (2008). Practical Hydro-sedimentology, $2^{\text {nd }}$ Edition International Publishers 599-603 pp.

[5] Ernest, C.I and E.U. Onweremadu (2016).Classification of Soil along ogochie River floodplain in Ngor-Okpala Imo State southeastern Nigeria. Journal of Soil Resources 2 (2): 76-83.

[6] Ezemonye, M.N and Emeribe, C.N, (2012). Rainfall Erosivity in Southeastern Nigeria. Ethiopian Journal of Environmental Studies and Management, 5(2): 1117.

[7] Gelagay, H.S. and A.S.Minale,(2016), Soil Loss estimation using GIS and remote sensing techniques: A cast of Koga watershed, Northwestern Ethiopia. International Soil and Water Conservation 4:126-136.

[8] Gunawan G., Dwita S., Herr, S., and W. Sulostiowemi, (2013), Soil Erosion Estimation based on GIS and Remote Sensing for Supporting Integrated Water
Resources Conservation Management, International Journal of Technology, 2 (3): 147-156.

[9] Ibeje, O.A (2016). Evaluation of Erodibility Status of Soils in Some areas of Imo and Abia States ofNigeria. International Journal of Earth and Environmental Sciences 1 (1): 1-9.

Michael, A.M and T.P. Ojha (2003), Principles of Agricultural Engineering, Volume II, Jain Publishers, New Dehli, India 3-436 pp.

[10] Nkheloane, T. Olaleye, A.O and R. Martin (2012). Spatial heteroenity of Soils physic-chemical Properties in contrasting wetland Soils in low agro-ecological zones of Lesotho. Soil Research Report 6:LESO 17-25 pp.

[11] Obi, M.E and F. N Salako (1995). Rainfall Parameters Influencing Erosivity in Southeastern Nigeria.Catena 24: 275-287.

[12] Okorafor, O.O, C. O Akinbile, A. J Adeyemo and C. C. Egwuonwu (2017). Determination of Rainfall Erosivity Index (R) for Imo State. American Journal of Engineering Research 6 (2):13-16.

[13] Okorafor, O.O, Akinbile, C.O and A.J. Adeyemo (2018). Determination of Soils Erodibility Factor $(\mathrm{K})$ for Selected Sites in Imo State, Nigeria. Resources and Environment 8(1): 6-13.

[14] Okorie, F.C, Nnaji, A, Chibo, C and E. Pat-Mbeno, (2012), Evidence of Climate Variability in Imo State of Southeastern Nigeria, Journal of Earth Science and Engineering 2: 544-553.

[15] Okoro, B.C. Uzoukwu, R.A and N.M. Chiemezie (2014).River basins of Imo State for sustainable Water resource management. Journal of Civil and Environmental Engineering 4 (1): 1-8.

[16] Oliveira, P.T.S, Wendland, E and M.A Nearing, (2012), Rainfall Erosivity in Brazil: A review. Catena 100: 139-147.

[17] Onwudike, S.U (2010). Effectiveness of Cow dung and Mineral fertilizers on Soil properties, nutrient intake, and yield of potato (Ipomea Batatas) in southeastern Nigeria.Asia Journal of Agricultural Research 4 (3): 148-154.

[18] Powlson, D. S., Gregory, P. J., Whalley, W. R., Quinton, J. N., Hopkins, D. W.,Whitmore, A. P., Hirsch, P. R., Goulding, K. W. T., (2011). Soil Management in Relation to Sustainable Agriculture and Ecosystem services. Food Policy Guidelines 36(2): 72-87.

[19] Renard, K. G., Foster, G. R., Weesies, G. A, McCool, D. K. and D.C Yoder (1997), Predicting soil erosion by water: A guide to conservation planning with the revised universal soil loss equation (RUSLE)USDA, Agriculture Handbook No.703, Washington DC 126$143 \mathrm{pp}$.

[20] Salako, F.N, Ghuman, B.S and R. Lal (1995).Rainfall Erosivity in South Central Nigeria.Soil Technology 7 (2): 79-88.

[21] Salako, F.K (2006). Rainfall Temporal Variability and Erosivity in sub-humid and Humid Zones of 


\section{International Journal of Engineering Applied Sciences and Technology, 2019 Vol. 4, Issue 6, ISSN No. 2455-2143, Pages 319-332 \\ Published Online October 2019 in IJEAST (http://www.ijeast.com)}

Southern Nigeria. Land Degradation and Development 17 (5): 541-555

[22] Salako, F.K. (2010), Development of Isoerodent maps for Nigeria from daily rainfall amount. Geoderma 156:372-378.

[23] Sanchez-Moreno, J.F, Mannaerts C.M and V. Jetten, (2013), Rainfall Erosivity Mapping for Santiago, Cape Verde.Geoderma 217: 74-82.

[24] Selemo, A.O, Ananaba, S.E, Nwagbara, J.O, Egejuru, V.E and V. Nwugha, (2012), Geostatistical Analysis of Rainfall Temperature and evaporation Data of Owerri for Ten years. Journal of Earth and Environmental Sciences 2: 195-205.

[25] Tey, H.S (2011), Soil Erosion Modeling using GIS and RUSLE for Cameron Highlands, Malaysia for Hydropower Development, School of Renewable Energy Studies, University of Iceland 29-45 pp.

[26] Udokporo, E, Anikwe, M.A.N and K.E. Chukwu (2015), Assessment and mapping of the Vulnerability of soils in Imo State Nigeria to erosion hazard using GIS. International Journal of Environmental Monitoring and Analysis 3 (5): 245-259.

[27] Ufot, U.O., Iren, O.B., and C.U. Chikere-Njoku (2016). Effects of Land use on Soil physical and chemical properties in Akokwa area of Imo State, Nigeria. International Journal of Life, Science and Scientific Research, 2 (3):1-6.

[28] Wang, B., Zheng, F., Romkas, M. J. M and F. Darboux,(2013), Soil Erodibility for Water Erosion; A Perspective and Chinese Experiences, Journal of Geomorphology, 187: 1-10.

[29] Yin, S., Xie, Y., Nearing, M.A and C. Wang (2007). Estimation of Rainfall Erosivity using 5-60 minute fixed interval rainfall data from China. Catena 70: 300-312. 